\title{
La consommation d'aliments traditionnels au Yukon, aux Territoires du Nord-Ouest et au Nunavut, étude Foodbook en 2014-2015
}

\author{
Vanessa Morton ${ }^{1 *}$, Anna Manore1, Nadia Ciampa², Shiona Glass-Kaastra², Matt Hurst², \\ Angie Mullen ${ }^{3}$, Jennifer Cutler ${ }^{1}$
}

\section{Résumé}

Contexte : Cet article présente un résumé descriptif de la consommation de divers aliments traditionnels (i.e. des aliments d'origine végétale et animale procurés localement dans la nature) par les résidents du Yukon (Yn), des Territoires du Nord-Ouest (T. N.-O.) et du Nunavut (Nt). Les données ont été collectées dans le cadre de l'étude Foodbook en 2014 et 2015.

Méthodes : L'étude Foodbook a été réalisée par téléphone sur une période d'un an. Les répondants ont été interrogés sur la consommation d'un large éventail de produits alimentaires au cours des sept jours précédents. Les habitants des territoires ont également été interrogés sur la consommation d'aliments traditionnels spécifiques à la région. Les données ont été pondérées afin d'élaborer des estimations territoriales de la consommation. Des données sur l'âge, le sexe, le lieu, le revenu et le niveau de scolarité ont également été recueillies.

Résultats : Le taux de réponse national pour le sondage Foodbook a été de 19,9\%. Au total, 1235 résidents des territoires ont participé à l'étude (Yn, $n=402, T$. N.-O., $n=458, N t$, $\mathrm{n}=375)$. La consommation d'aliments traditionnels au cours des sept jours précédents a été signalée par $77,5 \%, 60,7 \%$ et $66,4 \%$ des participants au Yukon, aux Territoires du Nord-Ouest et au Nunavut, respectivement.

Conclusion : Les réponses aux questions sur les aliments traditionnels posées en parallèle au questionnaire principal de l'étude Foodbook donnent un aperçu de la consommation d'aliments traditionnels au Yukon, aux Territoires du Nord-Ouest et au Nunavut.
Cette oeuvre est mise à la disposition selon les termes de la licence internationale Creative Commons Attribution 4.0

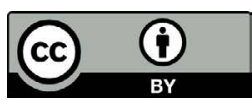

Affiliations

${ }^{1}$ Division de la gestion des éclosions, Agence de la santé publique du Canada, Guelph, ON

2 Division de la surveillance des maladies d'origine alimentaire et de la résistance aux antimicrobiens, Agence de la santé publique du Canada, Guelph, ON

${ }^{3}$ Ministère de la Santé, gouvernement du Nunavut, Iqaluit, NU

\section{${ }^{\star}$ Correspondance :}

vanessa.morton@canada.ca

Citation proposée : Morton V, Manore A, Ciampa N, Glass-Kaastra S, Hurst M, Mullen A, Cutler J. La consommation d'aliments traditionnels au Yukon, aux Territoires du Nord-Ouest et au Nunavut, étude Foodbook en 2014-2015. Relevé des maladies transmissibles au Canada 2021;47(1):35-42.

https://doi.org/10.14745/ccdr.v47i01a06f

Mots-clés : consommation alimentaire, aliments traditionnels, Nunavut, Territoires du Nord-Ouest, Yukon

\section{Introduction}

Il est important de disposer de données précises, complètes et actuelles sur la consommation alimentaire afin d'éclairer les programmes de santé publique et l'élaboration des politiques en matière de sécurité alimentaire et de nutrition, ainsi que les enquêtes sur les épidémies de maladies d'origine alimentaire.

Au Canada, les données sur la consommation alimentaire nationale sont disponibles grâce au sondage sur la santé dans les collectivités canadiennes mené en 2004 et 2015 (1,2). Malheureusement, ce sondage n'a pas permis de recueillir des données sur la consommation alimentaire au Yukon (Yn), aux Territoires du Nord-Ouest (T. N.-O.) ou au Nunavut (Nt).
Par conséquent, il existe peu de données provenant d'études nationales qui donnent un aperçu de la consommation alimentaire des habitants du Yukon, des Territoires du Nord-Ouest ou du Nunavut. L'étude Foodbook a été élaborée pour recueillir des données complètes sur la consommation alimentaire, utiles aux enquêtes sur les éclosions dans tous les provinces et territoires. L'étude Foodbook a utilisé un sondage téléphonique pour recueillir des données sur l'exposition alimentaire des résidents de tous les provinces et territoires. Le sondage Foodbook a été mené sur une période d'un an en 2014 et 2015 , avec une période de rappel de sept jours. Les données 
du sondage Foodbook ont depuis lors éclairé la réponse aux éclosions de maladies d'origine alimentaire au Canada en fournissant aux enquêteurs des valeurs de référence d'exposition alimentaire qui peuvent aider à la génération d'hypothèses (3-6).

En plus des aliments inclus dans le sondage national Foodbook, des aliments traditionnels supplémentaires ont été inclus spécifiquement pour les résidents du Yukon, des Territoires du Nord-Ouest et du Nunavut, comme recommandé par les représentants du gouvernement territorial. Les aliments traditionnels comprennent ceux qui sont récoltés sur la terre, dans la mer ou sous la glace, et peuvent inclure des mammifères terrestres tels que le caribou ou l'orignal, des mammifères marins tels que le phoque ou le morse, des poissons et des fruits de mer tels que l'omble chevalier, les palourdes et les moules, des oiseaux tels que les oies ou les lagopèdes, et des plantes telles que les baies (7-9). Les aliments traditionnels sont consommés au Yukon, aux Territoires du Nord-Ouest ou au Nunavut, ainsi que dans d'autres régions du Canada. Si les aliments traditionnels peuvent être consommés par des personnes d'origines culturelles diverses, la récolte, la préparation, le partage et la consommation d'aliments traditionnels soutiennent les liens avec le patrimoine culturel des peuples autochtones (10). En plus de favoriser les liens avec le patrimoine culturel, les aliments traditionnels sont souvent perçus comme étant plus savoureux, plus nutritifs et moins chers que les aliments achetés en magasin $(7,11,12)$. Les aliments traditionnels peuvent être obtenus par la chasse ou la cueillette, le partage entre membres de la communauté, la famille ou les amis, auprès d'organisations locales de chasseurs et de trappeurs, ou d'entreprises ou de particuliers qui vendent des aliments traditionnels $(7,11-13)$.

Comme pour les données de l'étude nationale Foodbook, les données sur la consommation d'aliments traditionnels ont été collectées dans le but de quantifier la prévalence de la consommation d'aliments traditionnels, des renseignements qui n'étaient pas disponibles lors des sondages nationaux précédents. En cas d'éclosion d'une maladie d'origine alimentaire au Yukon, aux Territoires du Nord-Ouest ou au Nunavut, ces données sur les fréquences de consommation des aliments traditionnels peuvent aider les enquêteurs à évaluer les aliments traditionnels spécifiques comme des expositions alimentaires potentielles d'intérêt. Ces données sont également potentiellement utiles pour les travaux sur la nutrition, la sécurité alimentaire, d'autres recherches sur la santé, ainsi que pour l'élaboration de politiques.

\section{Méthodes}

\section{Collecte de données}

Les données sur la consommation d'aliments traditionnels ont été collectées dans le cadre de l'étude nationale Foodbook. Foodbook est un sondage téléphonique menée auprès de la population dans toutes les provinces et tous les territoires canadiens, d'avril 2014 à avril 2015, avec des quotas mensuels pour assurer la représentativité au cours des différentes saisons. Les entretiens Foodbook ont été menés en anglais et en français dans toutes les provinces et territoires, et en anglais, français et inuktitut au Nunavut. On s'est adressé à des enquêtéssubstituts ont été utilisés pour les personnes n'ayant pas l'âge du consentement ou pour les personnes qui présentaient des restrictions d'ordre médical ou sur le plan des activités. Une traduction verbale était disponible sur demande pour d'autres langues selon les besoins. La conception de l'étude et la méthodologie d'échantillonnage pour l'étude Foodbook sont décrites en détail dans le rapport publié (14). En bref, une base de sondage pour les téléphones fixes et les téléphones portables a été utilisée pour sélectionner les répondants de chaque territoire. En plus des questions démographiques (e.g. le niveau de scolarité, le revenu du ménage, le code de région de tri d'acheminement), il a été demandé aux participants s'ils avaient consommé divers produits alimentaires spécifiques au cours des sept jours précédents. Les questions sur la consommation alimentaire portaient sur des articles appartenant aux catégories suivantes : fruits, légumes, herbes et épices, noix, viandes, poissons et crustacés, œufs, produits laitiers et aliments traditionnels. Une copie du questionnaire est disponible par l'entremise des données ouvertes (15). Les questions relatives à la consommation d'aliments traditionnels ont été posées uniquement aux participants du Yukon, des Territoires du Nord-Ouest et du Nunavut. Les questions relatives aux aliments traditionnels ont été rédigées et examinées avec les représentants des gouvernements territoriaux afin de s'assurer que les aliments prélevés dans la nature inclus reflètent les espèces animales et végétales disponibles ou consommées dans chaque territoire.

L'étude Foodbook a été examinée et approuvée par le Comité d'éthique de la recherche de Santé Canada et de l'Agence de la santé publique du Canada (CER 2013 0025) et par l'autorité d'éthique de la recherche en santé de Terre-Neuve-et-Labrador (HREB 13.238).

\section{Analyse statistique}

Les données ont été nettoyées, codées et analysées à l'aide de Stata 15.1 pour Windows (StataCorp LP, College Station, Texas, États Unis). Les valeurs manquantes, telles que les répondants refusant de répondre à une question, ont été retirées de I'analyse pour ces questions spécifiques. Les proportions ont été calculées en utilisant les poids de sondage, dont les détails sont décrits dans le rapport Foodbook (14).

Des variables composites décrivant des catégories d'aliments ont été créées sur la base d'une catégorisation biologique et d'une consultation avec les représentants des gouvernements territoriaux. Ces variables composites comprenaient tous les aliments prélevés dans la nature, le gros gibier, le petit gibier, la volaille sauvage, les mammifères marins, les poissons, les œufs sauvages et les plantes. Les variables composites ont été codées 
comme «oui » si la personne a déclaré consommer au moins un des articles et «non» si le répondant n'a déclaré consommer aucun des articles.

Un test de Wald ajusté a été utilisé pour évaluer les différences significatives de la variable composite «tout aliment prélevé dans la nature » entre les catégories de variables démographiques (i.e. l'âge, le niveau de scolarité, le revenu et le lieu). Les catégories faisant état de la plus forte proportion de consommation des aliments prélevés dans la nature ont servi de groupes de référence. Les comparaisons avec $p<0,05$ étaient considérées comme statistiquement significatives.

\section{Résultats}

Au total, 1235 personnes du Yukon $(n=402)$, des Territoires du Nord-Ouest $(n=458)$ et du Nunavut $(n=375)$ ont participé à l'étude Foodbook. La répartition géographique des répondants au sondage reflétait la répartition géographique des résidents au sein des territoires. Comme la localisation était fondée sur les trois premiers chiffres des codes postaux des participants, il n'était pas possible de séparer les données d'lqaluit du reste de la région de Qikiqtani au Nunavut. Quatre-vingt-dix-neuf pour cent des participants du Yukon et des Territoires du Nord-Ouest ont répondu à l'enquête en anglais, et le pour cent restant a répondu à l'enquête en français. Quatre-vingt-treize pour cent des participants du Nunavut ont répondu au sondage en anglais, six pour cent ont répondu à l'enquête en inuktitut et le pour cent restant a répondu à l'enquête en français.

La répartition par âge et par sexe des participants au Foodbook dans chaque territoire a été ajustée à l'aide de poids d'enquête pour être similaire à la répartition par âge et par sexe des populations de leurs territoires respectifs (tableau 1).

Tableau 1 : Caractéristiques démographiques, niveau de scolarité et revenu des répondants au sondage Foodbook pondérés par rapport aux données du recensement, Yukon, Territoires du Nord-Ouest et Nunavut, en 2014 et 2015

\begin{tabular}{|c|c|c|c|c|c|c|}
\hline \multirow[b]{2}{*}{ Caractéristiques démographiques, niveau de scolarité et revenu } & \multicolumn{2}{|c|}{ Yukon } & \multicolumn{2}{|c|}{$\begin{array}{l}\text { Territoires du Nord- } \\
\text { Ouest }\end{array}$} & \multicolumn{2}{|c|}{ Nunavut } \\
\hline & $\begin{array}{c}\text { Foodbook } \\
(\%)\end{array}$ & \begin{tabular}{|c} 
Population \\
recensée \\
$(\%)$
\end{tabular} & $\begin{array}{l}\text { Foodbook } \\
(\%)\end{array}$ & \begin{tabular}{|c|}
$\begin{array}{c}\text { Population } \\
\text { recensée } \\
(\%)\end{array}$ \\
\end{tabular} & $\begin{array}{c}\text { Foodbook } \\
(\%)\end{array}$ & $\begin{array}{l}\text { Population } \\
\text { recensée } \\
(\%)\end{array}$ \\
\hline \multicolumn{7}{|l|}{ Sexe $e^{a}$} \\
\hline Homme & 50,3 & 50,9 & 50,8 & 51,1 & 51,4 & 51,5 \\
\hline Femme & 49,7 & 49,1 & 49,2 & 48,9 & 48,6 & 48,5 \\
\hline \multicolumn{7}{|l|}{ Groupe d'âge $\mathrm{e}^{\mathrm{a}}$} \\
\hline $0-9$ ans & 11,5 & 11,3 & 14,8 & 14,1 & 22,9 & 22,8 \\
\hline $10-19$ ans & 12,4 & 10,9 & 15,1 & 13,2 & 19,3 & 18,0 \\
\hline $20-64$ ans & 67,1 & 67,6 & 64,3 & 66,2 & 54,4 & 55,7 \\
\hline $65+$ ans & 9,1 & 10,2 & 5,8 & 6,5 & 3,3 & 3,5 \\
\hline \multicolumn{7}{|l|}{ Éducation ${ }^{b, c}$} \\
\hline Inférieur au diplôme d'études secondaires & 9,5 & 10 & 28,4 & 18 & 45,6 & 49 \\
\hline Diplôme d'études secondaires & 17,4 & 21 & 14,3 & 22 & 5,4 & 15 \\
\hline $\begin{array}{l}\text { Certificat ou diplôme professionnel, d'un collège, d'un autre établissement non } \\
\text { universitaire/inférieur au baccalauréat }\end{array}$ & 41,1 & 39 & 28,7 & 37 & 17,2 & 23 \\
\hline Baccalauréat et certificats/diplômes supérieurs au baccalauréat & 31,2 & 29 & 27,0 & 23,0 & 29,7 & 13,0 \\
\hline Non communiqué & 0,8 & s.o. & 1,5 & s.o. & 2,2 & s.o. \\
\hline \multicolumn{7}{|l|}{ Revenu du ménage $^{d}$} \\
\hline Moins de $30,000 \$$ & 10,8 & 17,3 & 10,4 & 15,3 & 21,8 & 28,0 \\
\hline $30,000 \$-60,000 \$$ & 18,3 & 17,2 & 7,2 & 15,1 & 3,9 & 22,4 \\
\hline $60,000 \$-80,000 \$$ & 14,1 & 14,1 & 13,1 & 9,1 & 10,5 & 8,6 \\
\hline Plus de $80,000 \$$ & 49,0 & 51,5 & 52,2 & 60,5 & 42,9 & 41,0 \\
\hline Non communiqué & 7,8 & s.o. & 17,1 & s.o. & 21,0 & s.o. \\
\hline \multicolumn{7}{|l|}{ Lieu $^{e}$} \\
\hline Capitale territoriale & 80,3 & 77,1 & 45,6 & 48,0 & 61,9 & 52,3 \\
\hline En dehors de la région de la capitale & 19,2 & 22,8 & 50,2 & 52,0 & 38,1 & 47,3 \\
\hline Non communiqué & 0,5 & s.o. & 0,2 & s.o. & s.o. & s.o. \\
\hline $\begin{array}{l}\text { Abréviation : S.o., sans objet } \\
\text { Données sur la population territoriale, Statistique Canada. Tableau } 1710000501 \text { Estimation } \\
\text { o Données sur la population territoriale, Statistique Canada. Tableau } 3710011701 \text { Niveau de } \\
\text { autochtone, et population totale } \\
\text { c Question posée uniquement aux répondants du sondage Foodbook âgés de plus de } 25 \text { ans } \\
\text { Données territoriales, Statistique Canada, Enquête sur la santé dans les collectivités canadie } \\
\text { Données sur la population territoriale par code de région de tri d'acheminement, Statistique } \\
\text { Recensement de 2016. No de catalogue de Statistique Canada 98-402-X2016001. Ottawa. Pu }\end{array}$ & $\begin{array}{l}\text { épulation au } 1 \text { e } \\
132014 \\
13017 \text {. Tableau }\end{array}$ & $\begin{array}{l}\text { juillet, par âge e } \\
\text { on âgée de } 25 \text { à } 6\end{array}$ & $\begin{array}{l}\text { par sexe (donr } \\
\text { ans, populati }\end{array}$ & $\begin{array}{l}\text { nées de 2014) } \\
\text { on autochtone ho }\end{array}$ & s réserve, popul & lation non \\
\hline
\end{tabular}

Page 37 RMTC • Janvier $2021 \bullet$ volume 47 numéro 1 
Une plus grande proportion de participants au sondage Foodbook au Yukon, aux Territoires du Nord-Ouest et au Nunavut ont déclaré avoir un «baccalauréat ou un diplôme supérieur au baccalauréat» que les populations de recensement de leurs territoires respectifs (tableau 1). En outre, une proportion plus faible de participants au sondage Foodbook, par rapport aux populations recensées, dans les territoires a déclaré des revenus annuels des ménages inférieurs à 60000 dollars. Les poids de sondage n'ont pas été utilisés pour ajuster la répartition du niveau de scolarité ou du revenu annuel des ménages parmi les participants au sondage Foodbook.

La consommation d'un ou plusieurs aliments traditionnels au cours des sept jours précédents a été signalée par 66,4\% des participants au sondage Foodbook au Yukon, 60,7 \% aux Territoires du Nord-Ouest et $77,5 \%$ au Nunavut (tableau 2). Les répondants au sondage Foodbook âgés de 0 à 9 ans au Yukon et aux Territoires du Nord-Ouest étaient moins susceptibles de déclarer avoir mangé des aliments traditionnels au cours des sept jours précédents que ceux âgés de 20 à 64 ans. Aucune autre différence statistiquement significative n'a été notée entre les catégories d'âge (tableau 2). La proportion de répondants au sondage Foodbook ayant déclaré une consommation d'aliments traditionnels avec un revenu du ménage inférieur à 30000 dollars était significativement plus élevée que la proportion de répondants ayant un revenu du ménage supérieur à 80000 dollars dans les trois territoires.

II n'y a pas eu de différences significatives dans la variable composite «tout aliment traditionnel» rapportée parmi les catégories de la variable éducation au Yukon et aux Territoires du Nord-Ouest. En revanche, au Nunavut, la prévalence de la

Tableau 2 : Caractéristiques des répondants au Foodbook déclarant avoir consommé des aliments traditionnels au cours des sept jours précédents, Yukon, Territoires du Nord-Ouest et Nunavut, en 2014 et 2015

\begin{tabular}{|c|c|c|c|c|c|c|c|c|c|}
\hline \multirow{2}{*}{$\begin{array}{l}\text { Caractéristiques des } \\
\text { répondants }\end{array}$} & \multicolumn{3}{|c|}{ Yukon } & \multicolumn{3}{|c|}{ Territoires du Nord-Ouest } & \multicolumn{3}{|c|}{ Nunavut } \\
\hline & $\%$ & $95 \% \mathrm{Cl}$ & $p$ & $\%$ & $95 \% \mathrm{Cl}$ & $p$ & $\%$ & $95 \% \mathrm{Cl}$ & $p$ \\
\hline $\begin{array}{l}\text { Toute consommation d'aliments } \\
\text { traditionnels }\end{array}$ & 66,4 & $57,6-74,2$ & s.o. & 60,7 & $52,2-68,5$ & s.o. & 77,5 & $70,2-83,5$ & s.o. \\
\hline \multicolumn{10}{|l|}{ Sexe } \\
\hline Homme & 64,58 & $51,3-75,9$ & 0,6636 & 57,0 & $43,5-69,6$ & 0,3704 & 73,7 & $63,3-82,0$ & 0,2460 \\
\hline Femme $^{a}$ & 68,2 & $56,6-78,0$ & s.o. & 64,5 & $54,5-73,3$ & s.o. & 81,6 & $70,4-89,1$ & s.o. \\
\hline \multicolumn{10}{|l|}{ Groupe d'âge } \\
\hline $0-9$ ans & $53,3^{b}$ & $41,6-64,7$ & 0,0452 & $47,0^{\mathrm{b}}$ & $36,3-58,0$ & 0,0445 & 69,3 & $36,3-58,0$ & 0,3644 \\
\hline $10-19$ ans & 61,0 & $46,8-73,6$ & 0,3112 & 56,8 & $44,3-68,5$ & 0,4291 & 88,5 & $44,3-68,5$ & 0,1119 \\
\hline $20-64$ ans $^{\mathrm{a}}$ & 70,3 & $57,5-80,5$ & s.o. & 63,7 & $51,1-74,7$ & s.o. & 76,4 & $51,1-74,7$ & s.o. \\
\hline $65+$ ans & 61,6 & $50,0-71,9$ & 0,2877 & 71,4 & $57,7-82,1$ & $0,378^{c}$ & 88,6 & $57,7-82,1$ & 0,1619 \\
\hline \multicolumn{10}{|l|}{ Éducation } \\
\hline $\begin{array}{l}\text { Inférieur au diplôme d'études } \\
\text { secondaires }\end{array}$ & 86,9 & $68,1-95,3$ & 0,0709 & 73,1 & $38,5-92,2$ & 0,5064 & $94,5^{b}$ & $73,7-99,0$ & 0,0007 \\
\hline Diplôme d'études secondaires & 65,1 & $39,0-84,5$ & 0,9847 & 60,6 & $37,6-79,7$ & 0,9467 & 57,4 & $20,8-87,4$ & 0,9469 \\
\hline $\begin{array}{l}\text { Certificat ou diplôme professionnel, } \\
\text { d'un collège, d'un autre } \\
\text { établissement non universitaire/ } \\
\text { inférieur au baccalauréat }\end{array}$ & 72,8 & $52,1-86,8$ & 0,5599 & 58,8 & $41,0-74,5$ & 0,8246 & $83,7^{b}$ & $62,7-94,0$ & 0,0303 \\
\hline $\begin{array}{l}\text { Baccalauréat et certificats/diplômes } \\
\text { supérieurs au baccalauréat }^{\mathrm{a}}\end{array}$ & 64,8 & $43,1-81,7$ & s.o. & 61,6 & $42,8-77,5$ & s.o. & 55,9 & $36,0-74,0$ & s.o. \\
\hline \multicolumn{10}{|l|}{ Revenu } \\
\hline moins de $30000 \$$ & $84,9^{c}$ & $67,0-93,9$ & 0,0088 & $76,9^{\mathrm{b}}$ & $56,1-89,7$ & 0,0124 & $89,7^{b}$ & $73,3-96,5$ & 0,0137 \\
\hline $30,000 \$-60,000 \$$ & 69,5 & $50,5-83,6$ & 0,3981 & 53,6 & $35,2-71,1$ & 0,8884 & 68,4 & $39,6-87,8$ & 0,8512 \\
\hline $60,000 \$-80,000 \$$ & 67,5 & $48,3-82,2$ & 0,5159 & 51,3 & $24,8-77,1$ & 0,9609 & 67,1 & $38,6-86,9$ & 0,7802 \\
\hline Plus de $80,000 \$$ & 60,3 & $47,0-72,2$ & s.o. & 52,1 & $42,7-61,4$ & s.o. & 71,1 & $59,8-80,2$ & s.o. \\
\hline \multicolumn{10}{|l|}{ Lieu } \\
\hline Capitale territoriale ${ }^{b}$ & $62,0^{\mathrm{b}}$ & $51,6-71,4$ & 0,0018 & $47,8^{\mathrm{b}}$ & $37,8-58,0$ & 0,0039 & 73,1 & $63,1-81,3$ & 0,1031 \\
\hline $\begin{array}{l}\text { En dehors de la région de la } \\
\text { capitale }^{\text {a }}\end{array}$ & 84,2 & $72,1-91,6$ & s.o. & 71,0 & $57,9-81,3$ & s.o. & 83,8 & $72,8-90,9$ & s.o. \\
\hline
\end{tabular}


consommation d'aliments traditionnels était nettement plus élevée chez les personnes interrogées ayant un niveau de scolarité inférieur au diplôme d'études secondaires et chez celles ayant un certificat/diplôme professionnel, collégial ou non universitaire, par rapport aux personnes interrogées ayant un baccalauréat ou un diplôme supérieur au baccalauréat. La proportion de personnes interrogées consommant des aliments traditionnels était significativement plus élevée dans les régions du Yukon et des Territoires du Nord-Ouest situées en dehors des capitales Whitehorse et Yellowknife (tableau 2).

Les aliments traditionnels spécifiques consommés au cours des sept jours précédents varient selon les territoires (tableau 3). Dans au Yukon, les aliments traditionnels les plus souvent mentionnés étaient l'orignal $(46,0$; IC $95 \% 35,9-56,1)$, suivi des baies (28,3; IC $95 \%$ 18,3-38,4). Au Nunavut, le corégone était I'aliment prélevé dans la nature le plus souvent consommé $(25,8$; $95 \%$ IC 16,7-34,8), suivi du caribou $(22,0$; 95 \% IC 13,0-31,1) et de l'orignal $(19,8 ; 95 \%$ IC 13,0-31,1). Au Nunavut, le caribou $(57,2 ; 95 \%$ IC 48,6-65,9) et l'omble chevalier $(52,3 ; 95 \%$ IC 43,4-61,2) étaient les deux aliments traditionnels les plus souvent mentionnés. Les mammifères marins (e.g. le phoque, le morse, le béluga, le narval et la baleine boréale) ont été consommés par 43,2 \% (95 \% IC 34,1-52,3) des participants du Nunavut. La consommation d'aliments non prélevés dans la nature par les résidents du Yukon, des Territoires du Nord-Ouest et du Nunavut est indiquée dans le rapport Foodbook (14).

\section{Discussion}

L'étude Foodbook a recueilli des données sur la consommation alimentaire, y compris les aliments traditionnels, parmi les résidents du Yukon, des Territoires du Nord-Ouest et du Nunavut. Ces renseignements comblent une lacune dans les données nationales sur la consommation alimentaire. Au total, plus de $60 \%$ des répondants au sondage Foodbook dans chaque territoire ont déclaré avoir consommé un ou plusieurs aliments traditionnels au cours des sept jours précédents. Ces données montrent que les aliments traditionnels font partie du régime alimentaire de la majorité des résidents du Yukon, des Territoires du Nord-Ouest et du Nunavut. Les données présentées ici, ainsi que d'autres données recueillies dans le cadre des sondages Foodbook, peuvent être utilisées pour combler les lacunes dans les connaissances de ceux qui poursuivent des recherches dans les domaines de la sécurité alimentaire, de la sûreté alimentaire, des changements climatiques ou de la nutrition.

Étant donné la diversité du climat, des paysages, des populations et des pratiques culturelles dans les trois territoires, il est difficile de faire des comparaisons significatives entre les territoires en ce qui concerne les types d'aliments traditionnels mentionnés. Cependant, certaines tendances générales ont été observées concernant les résidents qui déclarent consommer des aliments prélevés dans la nature. Dans les trois territoires, le pourcentage
Tableau 3 : Répondants au Foodbook pondérés déclarant avoir consommé des aliments prélevés dans la nature au cours des sept jours précédents, Yukon, Territoires du Nord-Ouest et Nunavut, en 2014 et 2015

\begin{tabular}{|c|c|c|c|c|c|c|}
\hline \multirow{2}{*}{$\begin{array}{c}\text { Aliments prélevés } \\
\text { dans la nature }\end{array}$} & \multicolumn{2}{|c|}{ Yukon } & \multicolumn{2}{|c|}{$\begin{array}{l}\text { Territoires du } \\
\text { Nord-Ouest }\end{array}$} & \multicolumn{2}{|c|}{ Nunavut } \\
\hline & $\%$ & $95 \% \mathrm{Cl}$ & $\%$ & $95 \% \mathrm{Cl}$ & $\%$ & $95 \% \mathrm{Cl}$ \\
\hline Tout aliment traditionnel & 66,4 & $58,1-74,7$ & 60,7 & $52,4-68,9$ & 77,5 & $70,9-84,2$ \\
\hline Gros gibier & 51,9 & $42,2-61,6$ & 41,5 & $32,2-50,8$ & 59,4 & $50,8-67,9$ \\
\hline Caribou & 12,6 & $6,5-18,7$ & 22,0 & $13,0-31,1$ & 57,2 & $48,6-65,9$ \\
\hline Bœuf musqué & s.o. & s.o. & 3,4 & $0,6-6,2$ & 9,8 & $3,8-15,8$ \\
\hline Ours polaire & s.o. & s.o. & 0,0 & $0-0,01$ & 5,6 & $2,5-8,7$ \\
\hline Orignal & 46,0 & $35,9-56,1$ & 19,8 & $13,0-26,7$ & s.o. & s.o. \\
\hline Ours & 0,5 & $0-1,2$ & 0,9 & $0-2,5$ & s.o. & s.o. \\
\hline Bison & 12,1 & $6,0-18,3$ & 10,3 & $5,0-15,5$ & s.o. & s.o. \\
\hline Wapiti/cerf & 4,5 & $0-11,2$ & 2,5 & $0,1-4,9$ & s.o. & s.o. \\
\hline Mouton & 3,9 & $0-8,5$ & 1,3 & $0-3,0$ & s.o. & s.o. \\
\hline Volaille sauvage & 4,3 & $0,2-8,5$ & 6,6 & $3,2-10,1$ & 8,6 & $5,2-12,0$ \\
\hline Oie & s.o. & s.o. & 2,9 & $1,0-4,8$ & 6,6 & $3,6-9,7$ \\
\hline Canard & s.o. & s.o. & 2,7 & $0,8-4,7$ & 1,7 & $0,3-3,0$ \\
\hline Lagopède/tétras & 4,3 & $0,2-8,5$ & 3,7 & $0,8-6,6$ & 3,2 & $1,1-5,4$ \\
\hline Mammifères marins & s.o. & s.o. & 3,0 & $0,3-5,6$ & 43,2 & $34,1-52,3$ \\
\hline Phoque & s.o. & s.o. & 0,8 & $0-2,3$ & 28,9 & $20,3-37,5$ \\
\hline Morse & s.o. & s.o. & 0,0 & $0-0,01$ & 5,2 & $2,0-8,4$ \\
\hline Béluga & s.o. & s.o. & 2,2 & $0-4,4$ & 21,8 & $14,1-29,4$ \\
\hline Narval & s.o. & s.o. & 0 & $0-0,01$ & 11,6 & $6,8-16,4$ \\
\hline Baleine boréale & s.o. & s.o. & 0 & $0-0,01$ & 0,6 & $0-1,3$ \\
\hline $\begin{array}{l}\text { Petits mammifères } \\
\text { terrestres }\end{array}$ & 2,9 & $0-6,7$ & 5,3 & $1,9-8,6$ & s.o. & s.o. \\
\hline Spermophiles & 2,4 & $0-6,2$ & 0,0 & $0-0,01$ & s.o. & s.o. \\
\hline Castor/rat musqué & 0,0 & $0-0,01$ & 0,8 & $0-1,7$ & s.o. & s.o. \\
\hline Lapin & 2,9 & $0-6,7$ & 5,1 & $1,7-8,4$ & s.o. & s.o. \\
\hline Tout poisson & 16,8 & $8,5-25,0$ & 33,7 & $24,7-42,7$ & 53,9 & $45,0-62,8$ \\
\hline Omble chevalier & 1,9 & $0,5-3,3$ & 6,9 & $3,2-10,7$ & 52,3 & $43,4-61,2$ \\
\hline Corégone & 10,0 & $1,8-18,1$ & 25,8 & $16,7-34,8$ & 10,8 & $3,6-18,0$ \\
\hline Truite & 9,5 & $2,9-16,2$ & 7,9 & $4,3-11,5$ & 8,2 & $3,6-12,7$ \\
\hline Hareng & s.o. & s.o. & 0,5 & $0-1,0$ & 0,4 & $0-1,0$ \\
\hline Inconnu & s.o. & s.o. & 0,9 & $0-1,9$ & 0,2 & $0-0,3$ \\
\hline Saumon & s.o. & s.o. & 12,0 & $8,1-15,9$ & s.o. & s.o. \\
\hline Morue & s.o. & s.o. & 1,8 & $0,7-2,8$ & 5,9 & $2,6-9,2$ \\
\hline Brochet & 6,9 & $0-15,0$ & 4,5 & $1,5-7,4$ & s.o. & s.o. \\
\hline Fufs sauvages & s.o. & s.o. & 2,5 & $0-5,1$ & 4,9 & $1,5-8,2$ \\
\hline Fufs de canard & s.o. & s.o. & 1,1 & $0-2,6$ & 3,9 & $0,8-7,0$ \\
\hline Fufs d'oie & s.o. & s.o. & 0 & $0-0,01$ & 2,4 & $0,3-4,6$ \\
\hline Autres œufs sauvages & s.o. & s.o. & 1,7 & $0-3,9$ & 1,3 & $0-2,6$ \\
\hline Baies & 28,3 & $18,3-38,4$ & 14,4 & $8,5-20,3$ & 13,6 & $8,1-19,1$ \\
\hline Autres plantes & 6,8 & $2,0-11,7$ & 6,2 & $2,7-9,7$ & 5,8 & $2,4-9,1$ \\
\hline Algues marines & s.o. & s.o. & 6,8 & $2,2-11,4$ & 5,9 & $2,3-9,5$ \\
\hline
\end{tabular}


de répondants au sondage Foodbook qui consomment des aliments traditionnels augmente avec l'âge, à l'exception des personnes âgées de 20 à 64 ans au Nunavut. Cette association entre les aliments traditionnels et l'âge correspond aux résultats des sondages menés auprès des populations autochtones $(16,17)$. II semble également y avoir un lien entre le revenu et la consommation d'aliments traditionnels. Les raisons de ce lien ne sont pas claires, mais le coût des aliments achetés en magasin dans certaines communautés éloignées est assez élevé, de sorte que les participants ayant un revenu annuel plus faible peuvent compléter les aliments achetés en magasin par des aliments prélevés dans la nature bon marché. À l'inverse, d'autres études ont noté que les coûts liés à la chasse peuvent constituer un obstacle à la consommation d'aliments traditionnels (7).

Plusieurs études ont examiné la fréquence et la quantité de la consommation d'aliments traditionnels dans des régions et des communautés spécifiques du Yukon, des Territoires du Nord-Ouest et du Nunavut (tableau 4). Bien qu'il existe des points communs entre les méthodes utilisées dans ces études et dans celle du sondage Foodbook, les principales différences concernent les périodes de rappel et la méthode d'administration des sondages. Ces études précédentes diffèrent également de l'étude Foodbook par population cible. Les études figurant dans le tableau 4 se sont concentrées sur des communautés autochtones spécifiques. En revanche, l'étude Foodbook a recueilli des données pour l'ensemble de la population du territoire, indépendamment de l'appartenance ethnique. Ces différences méthodologiques rendent difficile la comparaison des résultats entre les études. Ces études, prises ensemble, peuvent plutôt être considérées comme complémentaires et permettent de mieux comprendre la consommation d'aliments traditionnels chez les habitants du Yukon, des Territoires du Nord-Ouest et du Nunavut.

Les principales limites de l'étude Foodbook sont énumérées dans le rapport Foodbook (14). L'une des limites spécifiques aux territoires était que l'enquête était administrée uniquement par téléphone. Cela a probablement affecté la représentativité des répondants à l'étude : alors que $99 \%$ des ménages canadiens ont accès au téléphone, dans les territoires, le nombre de ménages disposant d'un accès au téléphone est probablement inférieur à celui du sud du Canada (22). Une autre limite importante était le manque de données sur l'ethnicité. Ces données auraient fourni des renseignements sur le nombre d'autochtones inclus dans l'enquête et auraient aidé à comprendre les liens entre l'ethnicité et les habitudes de consommation alimentaire.

\section{Conclusion}

Dans l'ensemble, l'étude Foodbook en 2014 et 2015 a fourni un tableau complet de la consommation alimentaire au Canada et a inclus les territoires, qui n'avaient pas été inclus dans les études nationales précédentes. Il est également important de noter que l'étude Foodbook est l'un des rares sondages nationaux à offrir la possibilité d'être complétée en inuktitut, ce qui a pu permettre au sondage d'atteindre un segment de la population qui ne serait pas représenté autrement. Les données présentées ici peuvent fournir des renseignements pour soutenir la nutrition, la sécurité alimentaire, les sondages sur les épidémies et d'autres projets de recherche. L'ajout d'aliments traditionnels à d'autres études sur la consommation alimentaire devrait être envisagé dans d'autres zones géographiques, notamment dans les régions rurales et éloignées, afin de comprendre le rôle des aliments traditionnels dans le régime alimentaire des Canadiens.

\section{Déclaration des auteurs}
V. Morton et J. Cutler ont conçu l'idée de ce manuscrit. V. Morton et A. Manore ont élaboré la première ébauche. Tous les auteurs ont contribué à l'élaboration et à la révision de ce manuscrit et ont approuvé le projet final pour soumission.

\section{Tableau 4 : Sélection de sondages sur la consommation alimentaire menés au Yukon, aux Territoires du Nord-Ouest et au Nunavut}

\begin{tabular}{|c|c|c|c|c|}
\hline $\begin{array}{l}\text { Sondages sur la } \\
\text { consommation } \\
\text { alimentaire }\end{array}$ & $\begin{array}{l}\text { Étude sur la santé des } \\
\text { Inuits }(11,18,19)\end{array}$ & $\begin{array}{c}\text { Étude sur la santé des } \\
\text { enfants }(20,19)\end{array}$ & $\begin{array}{c}\text { Étude sur le Nunavut } \\
\text { de Sharma (21) }\end{array}$ & Étude Foodbook \\
\hline $\begin{array}{l}\text { Période de collecte des } \\
\text { données }\end{array}$ & Été 2007, été 2008 & Été 2007, été 2008 & $\begin{array}{l}\text { Septembre à décembre } \\
2006\end{array}$ & $\begin{array}{l}\text { Période d'un an en } 2014 \\
\text { et } 2015\end{array}$ \\
\hline Nombre de participants & 1569 & 338 & 188 & 1235 \\
\hline Critères de participation & $\begin{array}{l}\text { Adultes Inuits vivant dans } \\
\text { des communautés de la } \\
\text { région de peuplement Inuit }\end{array}$ & $\begin{array}{l}\text { Enfants Inuits de } 3 \text { à } 5 \text { ans } \\
\text { issus de } 16 \text { communautés } \\
\text { du Nt }\end{array}$ & $\begin{array}{l}\text { Inuits de plus de } 19 \text { ans, } \\
\text { non enceintes, dans quatre } \\
\text { communautés des T. N.-O. } \\
\text { et du Nt }\end{array}$ & $\begin{array}{l}\text { Résidents du Yn, des } \\
\text { T. N.-O. et du Nt; } \\
\text { sélectionnés pour répondre } \\
\text { aux quotas basés sur les } \\
\text { groupes d'âge }\end{array}$ \\
\hline Méthode d'enquête & En personne & En personne & En personne & Téléphone \\
\hline Période de rappel & 12 mois et 24 heures & Un mois & 24 heures & Sept jours \\
\hline
\end{tabular}

Abréviations : Nt, Nunavut; T. N.-O., Territoires du Nord-Ouest; Yn, Yukon 
A. Mullen est actuellement associé au ministère de la Santé et du Mieux-être de la Nouvelle-Écosse.

\section{Intérêts concurrents}

Aucun.

\section{Remerciements}

Les auteurs remercient le Centre des maladies infectieuses d'origine alimentaire, environnementale et zoonotique (Agence de la santé publique du (anada), la Division de la gestion des épidémies et la Division de la surveillance des maladies $d$ 'origine alimentaire et de la résistance aux antimicrobiens, les épidémiologistes provinciaux et territoriaux pour leur aide dans le cadre du projet Foodbook; le personnel de R.A. Malatest \& Associates Ltd. pour leurs entretiens d'experts. Nous remercions également J. Allen de Nunavut Tunngavik Incorporated pour la révision du manuscrit.

\section{Financement}

Ce travail est soutenu par l'Agence de la santé publique du Canada.

\section{Références}

1. Agence de santé publique du Canada. Rapport national sur la consommation alimentaire d'une journée : analyse des données du rappel alimentaire de 24 heures dans le cadre de l'Enquête sur la santé dans les collectivités canadiennes (ESCC), cycle 2.2, Nutrition (2004), et évaluation de la fréquence de consommation des aliments chez les Canadiens. Ottawa (ON) : ASPC; 2012. http://publications. gc.ca/site/fra/419161/publication.html

2. Santé Canada. Guide de référence pour comprendre et utiliser les données : Enquête sur la santé dans les collectivités canadiennes - Nutrition 2015 Ottawa (ON) : SC; 2017. https://www.canada.ca/fr/sante-canada/ services/aliments-nutrition/surveillance-alimentsnutrition/sondages-sante-nutrition/enquete-sant e-collectivites-canadiennes-escc/guide-referenc e-comprendre-utiliser-donnees-2015.html

3. Harvey RR, Heiman Marshall KE, Burnworth L, Hamel M, Tataryn J, Cutler J, Meghnath K, Wellman A, Irvin K, Isaac L, Chau K, Locas A, Kohl J, Huth PA, Nicholas D, Traphagen E, Soto K, Mank L, Holmes-Talbot K, Needham M, Barnes A, Adcock B, Honish L, Chui L, Taylor M, Gaulin C, Bekal S, Warshawsky B, Hobbs L, Tschetter LR, Surin A, Lance S, Wise ME, Williams I, Gieraltowski L. International outbreak of multiple Salmonella serotype infections linked to sprouted chia seed powder - USA and Canada, 2013-2014. Epidemiol Infect 2017;145(8):1535-44. DOI PubMed
4. Whitfield $Y$, Johnson $K$, Hanson H, Huneault D. 2015 Outbreak of Cyclosporiasis Linked to the Consumption of Imported Sugar Snap Peas in Ontario, Canada. J Food Prot 2017;80(10):1666-9. DOl PubMed

5. Hobbs JL, Warshawsky B, Maki A, Zittermann S, Murphy A, Majury A, Middleton D. Nuggets of Wisdom: Salmonella Enteritidis Outbreaks and the Case for New Rules on Uncooked Frozen Processed Chicken. J Food Prot 2017;80(4):703-9. DOI PubMed

6. Meghnath $K$, Hasselback P, McCormick R, Prystajecky N, Taylor M, Mclntyre L, Man S, Whitfield Y, Warshawsky B, McKinley M, Bitzikos O, Hexemer A, Galanis E; Outbreak Team. Outbreaks of Norovirus and Acute Gastroenteritis Associated with British Columbia Oysters, 2016-2017. Food Environ Virol 2019;11(2):138-48. DOl PubMed

7. Chan HM, Fediuk K, Hamilton S, Rostas L, Caughey A, Kuhnlein $H$, Egeland G, Loring E. Food security in Nunavut, Canada: barriers and recommendations. Int J Circumpolar Health 2006;65(5):416-31. DOI PubMed

8. Gouvernement de Nunavut - Département de santé. Guide alimentaire du Nunavut. https://livehealthy.gov.nu.ca/sites/ default/files/resource_attachments/FR_WEB_nunavut-foodguide.pdf

9. Nunavut Wildlife Management Board. Priest $\mathrm{H}$, Usher PJ. The Nunavut Wildlife Harvest Study. NWMB; 2004. https:// www.nwmb.com/inu/publications/harvest-study/1824156-nwhs-report-2004-156-0003/file

10. Lambden J, Receveur O, Marshall J, Kuhnlein HV. Traditional and market food access in Arctic Canada is affected by economic factors. Int J Circumpolar Health 2006;65(4):331-40. DOI PubMed

11. Nunavut Steering Committee. Egeland GM. 2007- 2008 Inuit Health Survey: Nunavut. Montreal (QC); McGill;2010. https://www.mcgill.ca/cine/files/cine/adult_report_nunavut. pdf

12. Inuvialuit Settlement Region Steering Committee. Egeland GM. 2007-2008 Inuit Health Survey: Inuvialuit Settlement Region. Montreal (QC): McGill; 2010. https://www.mcgill.ca/ cine/files/cine/adult_report_-_inuvialuit.pdf

13. Kuhnlein HV, Receveur O. Local cultural animal food contributes high levels of nutrients for Arctic Canadian Indigenous adults and children. J Nutr 2007;137(4):1110-4. DOI PubMed

14. Agence de la santé publique du Canada. Rapport Foodbook. Ottawa (ON) : ASPC; 2015. https://www. canada.ca/content/dam/canada/health-canada/migration/ healthy-canadians/publications/eating-nutrition/ foodbook-2015/alt/pub-fra.pdf

15. Données ouvertes. https://ouvert.canada.ca/fr/ donnees-ouvertes

16. Kuhnlein HV, Receveur O, Soueida R, Egeland GM. Arctic indigenous peoples experience the nutrition transition with changing dietary patterns and obesity. J Nutr 2004;134(6):1447-53. DOl PubMed 
17. Conseil de la radiodiffusion et des télécommunications canadiennes. Rapport de surveillance des communications 2018. Ottawa (ON) : CRTC; 2019. https://crtc.gc.ca/pubs/ cmr2018-fr.pdf

18. Saudny H, Leggee D, Egeland G. Design and methods of the Adult Inuit Health Survey 2007-2008. Int J Circumpolar Health 2012;71:19752. DOl PubMed

19. Kenny TA, Hu XF, Kuhnlein HV, Wesche SD, Chan HM. Dietary sources of energy and nutrients in the contemporary diet of Inuit adults: results from the 2007-08 Inuit Health Survey. Public Health Nutr 2018;21(7):1319-31. DOI PubMed
Child Health Survey 2007-2008. IPY; 2009. http://www. inuithealthsurvey.ca/pdf/Child_Inuit_Health_Survey_Aug_31. pdf

21. Sharma S, Cao X, Roache C, Buchan A, Reid R, Gittelsohn J. Assessing dietary intake in a population undergoing a rapid transition in diet and lifestyle: the Arctic Inuit in Nunavut, Canada. Br J Nutr 2010;103(5):749-59. DOI PubMed

22. Receveur O, Boulay M, Kuhnlein HV. Decreasing traditional food use affects diet quality for adult Dene/Métis in 16 communities of the Canadian Northwest Territories. J Nutr 1997;127(11):2179-86. DOI PubMed

20. Egeland GM; Qanuippitali Steering Committee Members. Qanuippitali? The International Polar Year Nunavut Inuit

\section{PRÉVENIR LA PROPAGATION DE LA COVID-19} 3258

MALADIE À CORONAVIRUS (COVID-19): COMMENT PRENDRE SOIN D'UNE PERSONNE ATTEINTE DE LA COVID-19 Ä LA MAISON CONSEILS AUX SOIGNANTS

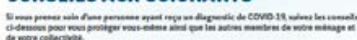

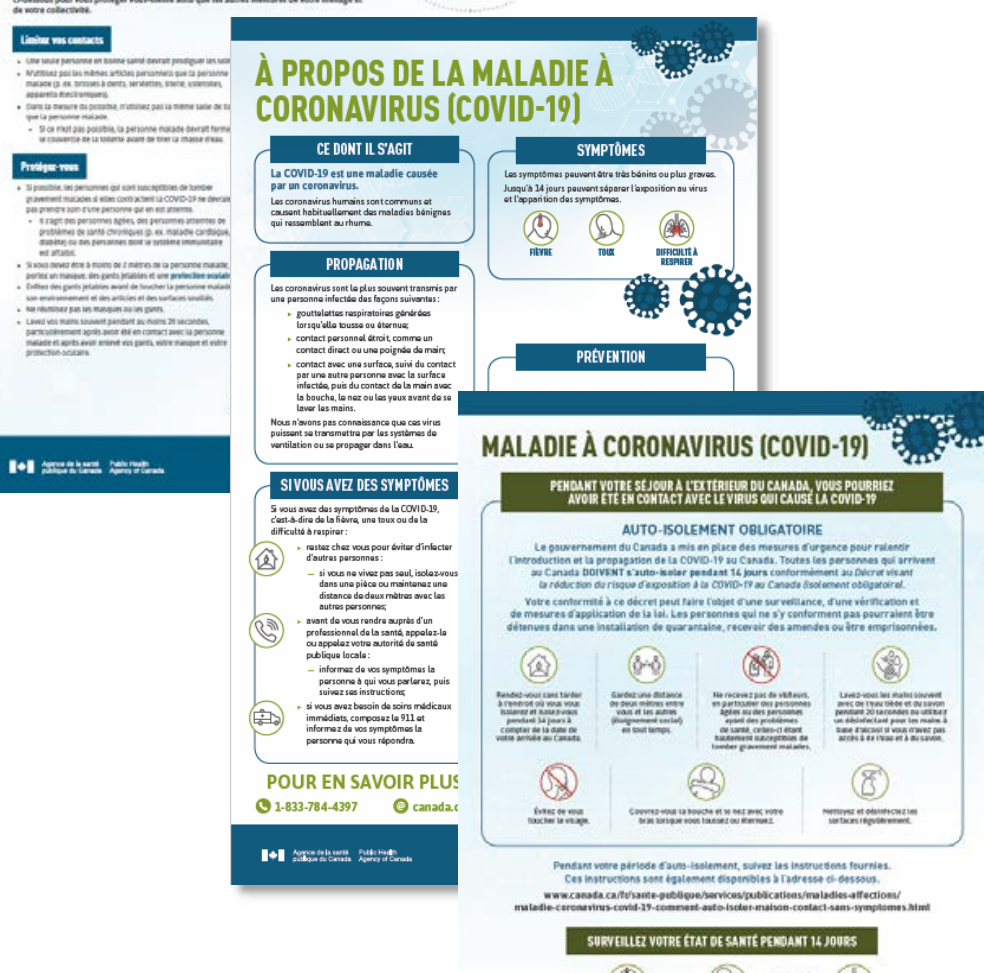

COVID-19

Ressources de sensibilisation

- Obtenez les dernières lignes directrices et ressources de sensibilisation

- Certaines ressources sont disponibles en plusieurs langues

\section{Visitez}

www.canada.ca/le-coronavirus 